\title{
Low Cost Autonomous Navigation and Control of a Mechanically Balanced Bicycle with Dual Locomotion Mode
}

\author{
Ayush Pandey, Subhamoy Mahajan, Adarsh Kosta, Dhananjay Yadav, Vikas Pandey, Saurav Sahay, \\ Siddharth Jha, Shubh Agarwal, Aashay Bhise, Raushan Kumar, Aniket Bhushan, Vraj Parikh, \\ Ankit Lohani, Saurabh Dash, Himanshu Choudhary, Rahul Kumar, Anurag Sharma, Arnab Mondal, \\ Chendika Karthik Sai, P N Vamshi \\ Indian Institute of Technology \\ Kharagpur, India $\mathbf{7 2 1 3 0 2}$ \\ Email: ayushpandey@iitkgp.ac.in
}

\begin{abstract}
On the lines of the huge and varied efforts in the field of automation with respect to technology development and innovation of vehicles to make them run on electric power and moreover autonomously, this paper presents an innovation to a bicycle. A normal daily-use bicycle was modified at low-cost such that it runs autonomously, while maintaining its original form i.e. the manual drive. Hence, a bicycle which could be normally driven by any human and with a press of switch could run autonomously according to the users needs has been developed.
\end{abstract}

Index Terms - road vehicles, bicycles, electric vehicles, autonomous bicycle

\section{INTRODUCTION}

The paper is divided into three sections broadly, viz. the mechanical design, the control and planning and finally the actual implementation of the bicycle along with the limitations and applications of the product. The point kept in mind throughout the design process was that the full cost of the modifications made to the bicycle should be low and the electrical and computational complexity as well should be minimized so as to lead to robust product. These requirements were met to a great extent as shall be shown in the following sections.

Any autonomous vehicle along with all its advantages of reducing human effort and better repeatability etc. carries some disadvantages which are very well known and are majorly the reasons why autonomous vehicles arent yet on the roads and are far from daily usability. The biggest advantage of this autonomous bicycle, (named i-Bike) is that it takes the best of both the worlds, the robustness and fail-safe properties of manual vehicles along with the low human effort, userfriendliness and advanced-innovative tools available in an autonomous vehicle. The i-bike successfully achieves that. Added to this, during the design process of the i-Bike it was realized that achieving a full fledged autonomous bicycle is a humongous task (pertaining to its unstable nature) and one that requires great efforts to achieve robustly. Hence, the identity of the bicycle wasnt sacrificed by making only as few modifications (reversible ones) as possible at low-cost.
The motivation to build this product comes from the fact that its always difficult and time consuming to retrieve ones bicycle from the parking stand (for eg. in a bike-share system). It is even worse for someone who has a disability or is a differentlyabled person, people who can drive a bicycle but face a lot of difficulty in getting it back from the parking zone, which usually turns into a disorganized area, especially in a country like India. The i-bike solves this problem along with being user friendly as described in the last section and has many other applications as well. It has certain other features as well such as tracking through GPS; wireless GSM based control and vehicle security applications. In essence, the modifications brought in serve the purpose to a great extent and are quite robust and feasible both in terms of its cost and durability. The initial two sections describe the technical details of the modifications made to the bicycle, mechanically (first section) and in control and motion planning (second section).

\section{BACKGROUND}

The problem of autonomous stabilization of bicycles has been an area of research for many years and a lot of methods have been proposed. One of the most common methods of balancing a bicycle is the use of inverted pendulum model. In this method, the balancer (inverted pendulum) configuration is changed according to the states of motion and position of the bicycle elements. Experimental study of balancing an autonomous bicycle with a balancer has been reported in [2] and [3]. Stabilizing the bicycle with additionally controlling the steering has much better performance and has been reported in [4] and [5]. Further works have been reported which involve acrobatic maneuvers of a bicycle using a new balancer configuration [1]. In all of the reported experimental setups, the balancer is controlled by a motor, thus constantly consuming energy and insensitive to abrupt changes in the configuration of the bicycle which will require faster motors with higher torques. However, this paper presents a method to attain the same with ease using a mechanically implemented control which stabilizes the roll of the system (passive stabilization). 
The research focuses on the novel system design to achieve the stability mechanically and to convert the bicycle into a drive by wire system with proper modifications to the braking and driving. The uniqueness of the design is that the bicycle doesn't lose its normal operation and can be operated in a dual locomotion mode (i.e. can be switched back with ease to its normal manual operation from autonomous mode).

\section{MEChaniCAL DESIGN}

The mechanical design of each and every component is such that it only adds onto the existing structure of the bicycle and any modification does not interfere with riding capabilities of a normal user or rider. The volume a rider may occupy was also kept in mind while designing each and every structure.

\section{A. Drive Mechanism}

Driving Mechanism [6] refers to the complete mechanism designed for the translation of the bicycle. In autonomous mode, the drive mechanism enables it to translate both in forward and backward directions. There are various ways in which driving can be achieved in vehicles. Electric motors are the most commonly used actuators due to their easy availability, simple setup and cheap nature. The i-Bike uses electric motors as well. Power transmission can be achieved using various methods like chain drive, shaft drive, wire rope and pulley drive. Hydraulics can also be used as well for power transmission. But the most economical and viable option was the chain and sprocket mechanism which was used in the iBike.

A normal bicycle has a free wheel on the rear hub shell and there is ample space for a motor driven chain-sprocket mechanism to be installed for automation. The motor was placed on a base supported on the rack of the bicycle (as shown in Figure) because it provides a firm and rigid base reducing the need to make design changes to the bicycle.

The freewheel was modified which works as a sprocket. To provide for both autonomous and manual cycling modes, a mechanism was provided for engaging/disengaging the motor from chain-sprocket assembly by simply sliding and locking it. Disengaging/engaging mechanism was chosen such that the cost of manufacturing is low and is easy to use. Proper SolidWorks designs were made and tested for strains before fabricating on the bicycle. Two shafts were designed based on lock and key mechanism mentioned. One of the shaft was attached to the motor's shaft and another part was attached to modified freewheel supported by two self-aligning ball bearings. The use of self-aligning ball bearings reduces high accuracy needs which is otherwise very important to align the motor's shaft with the modified freewheels shaft. The mechanism is engaged when the two components move towards each other and unlocked when moved away from each other. To allow for such a movement, motor is provided with a base which slides on the main base. The shaft attached to the motor was of hexagonal shape and the shaft on the sprocket had its counterpart.

The shape of the shaft was chosen such that the engaging process is easy and there would be less wear in the shaft. Hexagonal shape was chosen so that cyclist could rotate the shaft by 30 degrees maximum ( $15 \mathrm{~cm}$ forward or backward) for engaging the motor. The material used for the shafts was mild steel and the lock and key part was flame hardened to reduce wear and tear.

\section{B. Steering Mechanism}

Efficient steering mechanism [7],[8] is required to control the bicycle autonomously and its design plays a very major role in position control as it controls the direction in which the bicycle would move.To steer the bicycle, the motor needs to overcome the torque on the stem due to gravity and centrifugal torque, gyroscopic effects and momentum induced torque. We calculate the required motor torque using the following equation.

$$
\text { Torque }=\Delta_{\text {scalar }} \theta_{f} \sin (\phi)+\sigma \cos (\phi) \frac{A}{L} M g
$$

where, $\Delta_{\text {scalar }}$ is scalar value of trial vector, $\theta_{f}$ is the lean axis of the frame, $\phi$ is the steering axis angle, $\sigma$ is the turn angle of the handle bar, $L$ is the distance between the wheel hubs and $A$ is the horizontal distance between the rear wheel hub and the centre of mass. The required torque using Eq. (1) comes out to be $2.7 \mathrm{Nm}$.

A servo motor matching required torque was used to steer the bicycle which was mounted parallel to the head tube. A chain sprocket mechanism was used for power transmission. A sprocket freely moves below the headset and can be tightened using a lever welded to headset. On tightening the sprocket motor is engaged with the stem of the bicycle due to high friction and to disengage the motor sprocket is loosened. The motor was mounted using clamp attached to top tube and parallel to the head tube.

\section{Braking Mechanism}

Three mono pivot breaks were used for braking, two of which were at their normal position which were manually operated and the third was attached on the front wheel opposite to the normal position of manual brake.

Autonomous braking was controlled by a DC motor which rotates a disk on which a wire gets wound up. The motor when switched on pushes the brakes pads towards the wheel, but prolonging the same may damage the motor.

\section{Balancing Mechanism}

Usually bicycles are balanced with electronic feedback control; in this bicycle the aim was to reduce the already complex system by using mechanical feedback control. There can be many such control systems; we chose to use a torsional spring and damper system for the same.

A pair of balancing wheels was used which remain in contact with the ground even when bicycle is upright. They were attached on the axle of rear wheel hub. A normal set of training wheels would not allow the bicycle to lean while taking turns. A balancing wheel includes a torsional spring on which a freely rotating wheel was mounted. 


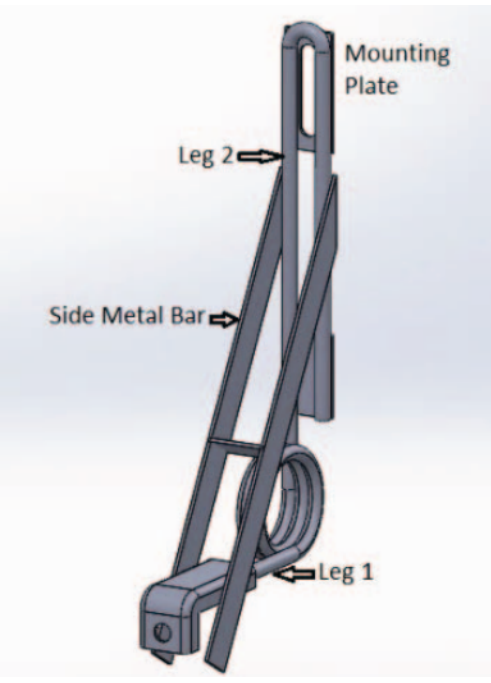

Fig. 1. Spring balancer model

Spring Design: Both springs were helical torsion spring with right handed helical loop for left spring and left handed helical loop for right spring. If there is no restriction, leg 1 of spring acts as a cantilever beam and vibrates about vertical axis. The two side metal bars restrict the vibration of the spring but allow winding of spring. A plate with slot was welded on other leg of spring. The rear wheel hub bolt come through this slot and whole assembly was tightened by using a nut. The slot on plate allows adjusting height of balancing wheel. Calculation of Spring Constant: Considering space constraints the mean coil diameter of spring was kept at $5 \mathrm{~cm}$. The radius of rear wheel of bicycle was $30 \mathrm{~cm}$ and of trainer wheel was $6 \mathrm{~cm}$. Therefore, length of leg $1\left(l_{1}\right)$ is given by:

$$
l_{1}=30-6-2.5=21.5 \mathrm{~cm} \text {. }
$$

If the bicycle tilts in one direction while turning or due to some obstacle; it should be able to regain vertical position all by itself. Considering a situation where the bicycle is tilted and makes an angle $\theta$ with vertical. Therefore, spring also winds by an angle of $\theta$.

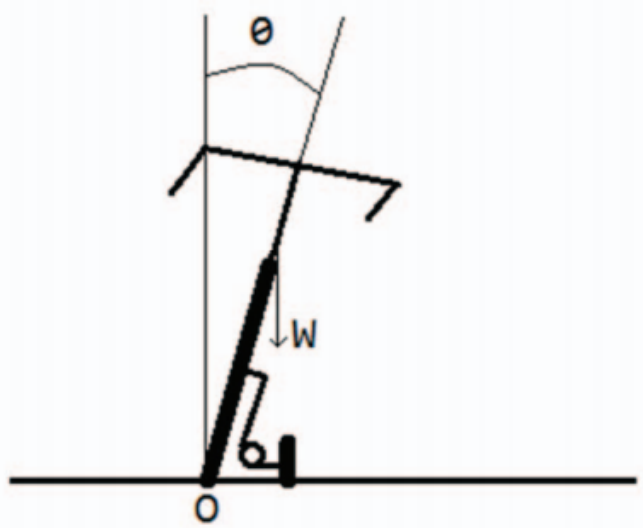

Fig. 2. Free body diagram for torque calculation
Torque due to weight of bicycle about point $\mathrm{O}$ is given by the following equation.

$$
T_{w}=m g h \sin (\theta)
$$

where $m$ is the mass of the bicycle without one balancing wheel (25 kg in our case), $g$ is acceleration due to gravity and $h$ is the height of the center of mass of the bicycle from ground in the vertical position (which is $0.6 \mathrm{~m}$ in our case). The torque due to spring about point $\mathrm{O}$ is then given by

$$
T_{s}=\frac{k R}{l_{1}}
$$

where $k$ is the spring constant (in $\mathrm{Nm} /$ radian), $R$ is the radius of the rear wheel (equal to $0.3 \mathrm{~m}$ in our case) and $l_{1}$ is the length of leg 1 ( $0.215 \mathrm{~m}$ for our bicycle design).

For the bicycle to regain vertical position, there must be a net counterclockwise torque about point $\mathrm{O}$ for every value of $\theta$ greater than 0 .

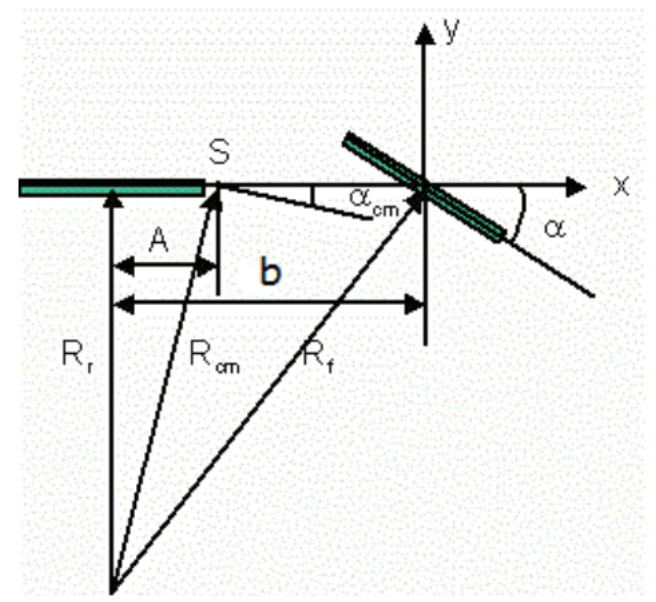

Fig. 3. Balancing Wheel Analysis, where $b$ is the wheel base $(1 \mathrm{~m}) ; A$ is the horizontal distance of the center of mass of the bicycle from the rear wheel axle $(.4 \mathrm{~m})$; is steering angle and $R \mathrm{~cm}$ is the radius of the turn of the center of mass of bicycle

$$
\begin{aligned}
T_{s}-T_{w} & >0 \\
T_{s} & >T_{w} \\
\frac{k \theta R}{l_{1}} & >m g h \sin (\theta) \\
\frac{k R}{m g h l_{1}} & >\frac{\sin (\theta)}{\theta}
\end{aligned}
$$

As $\theta \rightarrow 0$ we have, $\frac{\sin (\theta)}{\theta} \rightarrow 1$. So, we can write

$$
\frac{k R}{m g h l_{1}}>1
$$

We get the value of $k$ on substitution

$$
k>117.7 \mathrm{Nm} / \mathrm{rad}
$$


Now from Fig(3) we can write

$$
R_{c m}=\sqrt{A^{2}+\left(\frac{b}{\tan (\alpha)}\right)^{2}}
$$

Using the above relation we conclude that the maximum steering angle should be $30^{\circ}$. Hence, the smallest possible value of $r$ would be $1.78 \mathrm{~m}$. Now, we can write the torque of the centrifugal force about point $\mathrm{O}$, while taking a turn as follows

$$
T_{c}=\frac{m v^{2} h \cos (\theta)}{r}
$$

where $m$ is the mass of the bicycle, $v$ is the velocity of the bicycle and $r$ is the turning radius. Although the maximum possible velocity of bicycle is $5 \mathrm{~m} / \mathrm{s}$, while turning it would be brought down to $2 \mathrm{~m} / \mathrm{s}$ to reduce the centrifugal force. Hence, to obtain the maximum value of $T_{c}$ we would use the maximum value of velocity $(2 \mathrm{~m} / \mathrm{s})$ and the minimum turning radius to be equal to $1.78 \mathrm{~m}$. At this condition, the bicycle would not tilt by more than $10^{\circ}$. Therefore, at $\theta=10^{\circ}$ the net torque about point $\mathrm{O}$ would be equal to zero.

$$
\begin{aligned}
T_{s} T_{w} T_{c} & =0 \\
T_{s} & =T_{w}+T_{c} \\
\frac{k R}{l_{1}} & =m g h \sin (\theta)+\frac{m v^{2} h \cos (\theta)}{r}
\end{aligned}
$$

Substituting for the parameters, we get $k=4.9 \mathrm{Nm} /$ degree, using

$$
k=\frac{d^{4} E \pi}{64 \times 180 D N_{a}}
$$

where $d$ is the diameter of the spring wire, $E$ is the modulus of rigidity of stainless steel (180GPa), $D$ is the mean coil diameter $(5 \mathrm{~cm})$ and $N_{a}$ is the equivalent number of active turns

$$
N_{a}=N_{b}+\frac{l_{1}+l_{2}}{3 \pi D}
$$

where $N_{b}$ is the number of body turns (2.25). Substituting the values and equating to $k=4.9 \mathrm{Nm} / \mathrm{deg}$, we get $d=1.47 \mathrm{~cm}$.

\section{Calculation of maximum safe compression of spring}

Minimum tensile strength of spring $S_{u t}=\frac{A}{d m}$. For stainless steel wire, $A=2911 \mathrm{MPa}-\mathrm{mm}^{m}$ where $m=.478$. Torsional yield strength $S_{y}=.61 S_{u t}=491.4 \mathrm{MPa}$. Bending stress for round wire torsion spring is given by the following equation

$$
\sigma=\frac{32 K_{i} M}{\pi d^{3}}
$$

Where $K_{i}$ is the bending stress correction factor and $M$ is the moment of the force acting on the spring

$$
K_{i}=\frac{4 c^{2}-c-1}{4 c(c-1}
$$

where $c$ is the spring index, which is equal to $D / d$. At maximum compression $\sigma=S_{y}$.

$$
M=\frac{\pi d^{3} S_{y}}{32 K_{i}}=119.7 N m
$$

Therefore, the maximum safe compression angle is given by

$$
\theta=\frac{M}{k}=24.4^{\circ}
$$

which is greater than $10^{\circ}$

A shorter length of leg 2 will increase compressive stress on trainer wheel which was made of plastic but longer length will increase its probability of hitting obstacles. So to optimize the length of leg 2, a stress analysis of trainer wheel was performed in SolidWorks. The moment of normal force acted

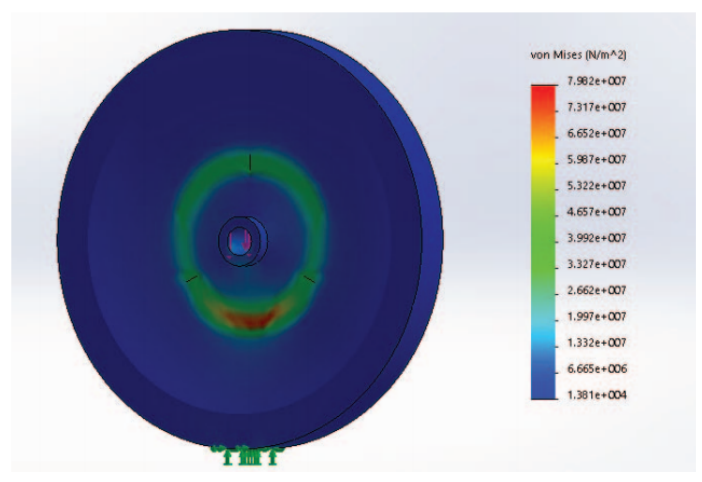

Fig. 4. Stress analysis of the trainer wheel

by ground on trainer wheel balances the moment of spring. So, maximum normal force will occur when spring is compressed at maximum angle, which is $10^{\circ}$

$$
N_{\max }=\frac{M_{\max }}{l_{2}}
$$

where $M_{\max }$ is the maximum value of moment $(641.5 \mathrm{~N})$ and $l_{2}$ is the length of leg 2 .

Trainer wheel was modeled in SolidWorks and High Density Polyethylene (HDPE) material was assigned. Stress analysis was performed at different $\mathrm{Nmax}$ by increasing values of 2 until sufficient factory of safety was obtained. At ${ }_{2}=.11 \mathrm{~m}$, Factor of Safety of 2 was obtained. Hence length of leg 2 was kept at $.11 m$.

\section{Control and Motion Planning}

The mechanical modifications made in the bicycle need to be actuated properly and the required peripherals were added to complete the automation process. The complete process is divided into various parts described in detail as subsections.

\section{A. Drive Control System}

The translation motion of the i-Bike, both forward and backward, and hence the velocity control is achieved using a feedback control system. The actuators used in the control 
system were two high torque DC motors [9]. The sensor giving the velocity feedback in the form of counts per revolution is the rotary encoders placed around the motor shaft. The control was achieved using a PID controller algorithm [10] implemented on an electronics chip.

The steer motor was coupled using a sprocket and chain mechanism directly to the main handle of the bicycle and the drive motor was coupled using a similar mechanism to an extension to the back wheel of the bicycle. As mentioned earlier, the bicycle can move using human power and also can be motor driven. So for achieving this, an engagingdisengaging mechanism was added to the drive motor for control method chosen.

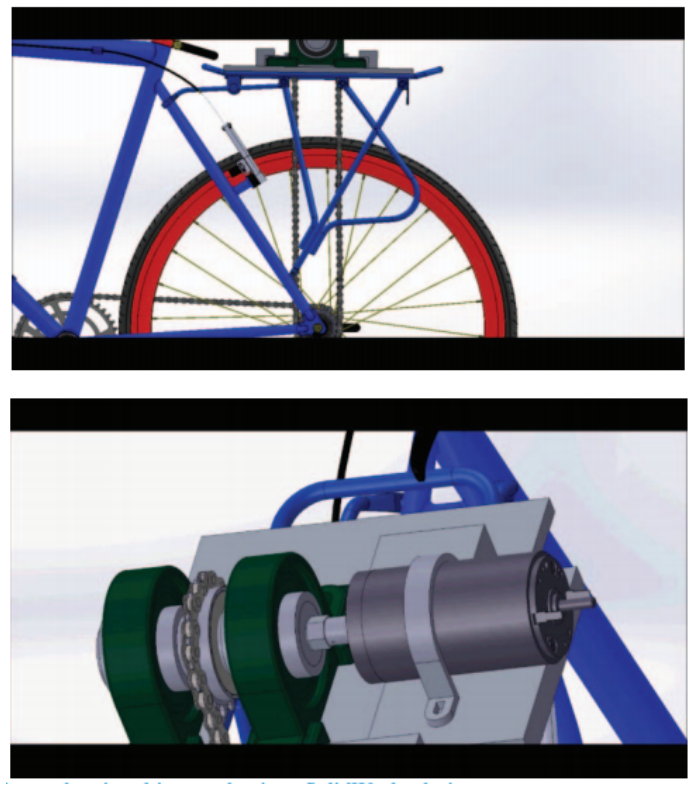

Fig. 5. Drive control mechanism

The motor was driven using a pulse given by the microcontroller, the Arduino having ATmega 2560 chip on which the control algorithm was implemented. The frequency of the PWM decides the speed to which the motor is sped.

The motor requires quite a high amount of current at full load (7 A) and this was achieved using lightweight but high capacity Lithium Polymer (LiPo) batteries of $2200 \mathrm{mAH}$. The motors were successfully able to do both operations, given their high amount of advertised torque $(120 \mathrm{Kg}-\mathrm{cm})$. The battery lasts through about 2 hours on a single charge. This may be improved further by using motors with lower current rating.

Tests were performed on the mechanisms for testing for any kinds of slipping due to mechanical fault and results were satisfactory in case of the drive motor, but the steer motor encountered a lot of slipping in a particular direction, which was corrected by an LED-LDR pair (an Opto-Coupler).

For the localization of the bicycle, a basic form of odometry [11], [12] was also implemented using simple geometric formulae and using data of encoders on the motor, which were

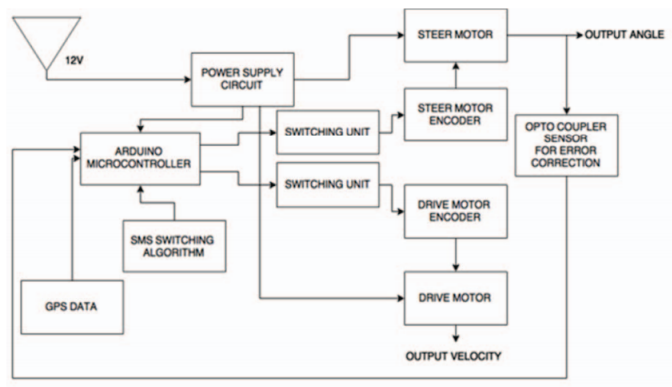

Fig. 6. Flow chart depicting control system schematic

correct to an error of 5-7can be perfected by using encoders on the wheel rim and calculating the distance using real world data, incorporating all kinds of mechanical errors. This would make the path planning code work more accurately. For now, The i-Bike relies on the motor encoder assuming that the control mechanisms inside work perfectly. In future implementations, external control systems might be used to incorporate errors.

\section{B. Localization}

Magnetometers were used on top of the bicycle for yaw measurement during the motion. Since magnetometer provides the angle with respect to the geometric north, Yaw was measured every time assuming linear variation of magnetometer angle change with the yaw for a very small angle.

For a more precise localization of the bicycle [11], along with Yaw measurement for orientation, wheel odometry for the drive motor was implemented. These calculations lead to the position of the bicycle known to the controller at every instant with a fair deal of accuracy. The counts from the encoders of the drive and steer motor help us to calculate angle and distance travelled by the bicycle in discrete time steps using the model that it rotates in a circle for every small time interval.

\section{Steer Angle Measurement and Control}

A dc motor was used to actuate the steering control system in the bicycle. The complete control system for the steering was achieved in a complex way incorporating various innovations. This was needed since the velocity control of the steer drive is not sufficient in itself for the accurate steering control. The main reason for inaccuracies is slippage between the motor axle and sprocket in the mechanical design. Hence, the need for a closed loop control on top of the internal encoder based velocity feedback control was realized using a potentiometer fixed on the drive using an L-clamp [13].

During testing, the control system was further improved upon since the central position of the bicycle was still in doubt and incorrect results were being obtained. Hence, a custommade encoder was designed and placed on the mudguard of the bicycle. The encoder was realized in the form of an IR sensor, called the MOC sensor. A flap was designed which was placed judiciously such that every time the wheel crossed the central position a signal was received and we could eliminate 


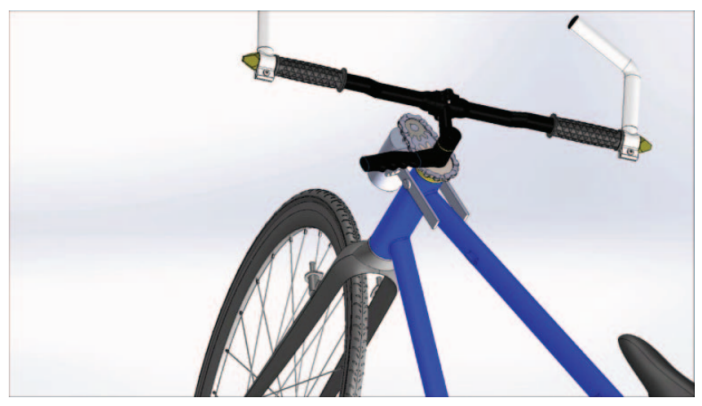

Fig. 7. Steering mechanism design

all the errors accumulated in that run (and hence nullify all such errors). This lead to a very accurate steer angle control mechanism.

The steering microcontroller receives the data from the drive microcontroller which acts as a path planner for the bicycle, giving it the steering angle to rotate, after calculations and estimations suggested by the Obstacle Detection module.

On encountering an obstacle, the obstacle avoidance module would be activated instantly (prioritized over others) and its data would be overridden on the steering control so that the safety is maintained as a first priority. In future, there is scope to make the algorithm for obstacle avoidance and planning more robust for better practical application.

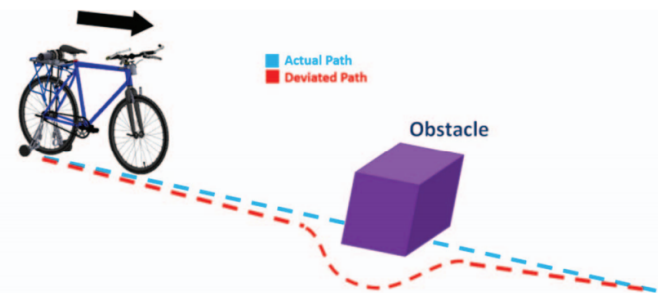

Fig. 8. Motion planning algorithm

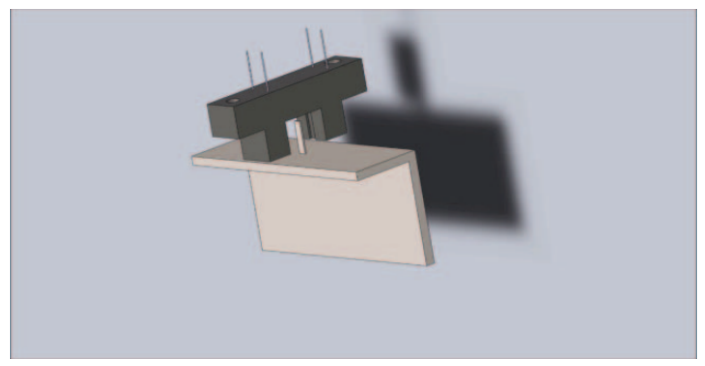

Fig. 9. Encoder working mechanism to reduce errors in steering control

\section{Obstacle Detection and Avoidance}

Two ultrasonic SONARs with a range of 2-400 $\mathrm{cm}$ were used for obstacle detection in which time of flight of the ultrasonic wave is determined and hence obstacle distance and angle (measured because of the position of the sensor).

The i-Bike uses 2 SONARs inclined at an angle of 10 degrees with vertical in same horizontal plane. The SONARs detect obstacles on either side of the bicycle with overlapping cones for also detecting obstacles in front of bicycle, covering a total obstacle detection cone of 45 degrees. In this way, obstacle detection with a considerable field of view was achieved. After the detection of an obstacle, the control was shifted to the obstacle avoidance module which was implemented on the microcontroller driving the bicycle.

\section{E. Autonomous Braking}

A low rpm dc motor with appropriate torque (to pull the brake wire) was used as the braking actuator. The braking wire was attached to the axis of the dc motor and the other end was attached to the bicycle rear wheel braking mechanism used in normal bicycles. A proper motor angle range was chosen to brake the front wheel to make sure that the braking shoes maintain a constant range of braking force required to stop the bicycle. Currently, the system implements braking as an open loop system, improvements could be made upon this design to make the braking closed-loop by detecting and choosing when to brake. Additionally, along with this braking system the drive motor on the back was capable on itself to slow the bicycle down (or even move it backwards!). Though, since currently the bicycle demonstrates very low speeds such as $5 \mathrm{~m} / \mathrm{s}$ or even less, braking is not much of a concern. But, the arrangement in place ensures for the autonomous braking whenever needed.

\section{F. User-friendly Interface and Other Features}

Autonomous Navigation in the bicycle was achieved by localizing it with respect to its initial position using global positioning system (GPS) and then it could be controlled using a SMS (received from the user) which could either contain destination GPS co-ordinates or relative position of the destination with respect to its initial position. This has been discussed in [1]. SMS communication was used to communicate between user and bicycle making it considerably simple and user friendly. [2] explains more in detail on this fact.

To facilitate this SMS based system in the bicycle, GSM SIM 900 module was interfaced with the controller to receive/send messages regarding the target location which bicycle needs to achieve or any other control actions. The current location of the bicycle would be determined by the GPS module.

\section{G. Online Tracking of the Bicycle}

This significant feature in the bicycle was achieved using GPRS technology available in the GSM SIM 900 module. A GSM modem containing a SIM card of any valid service provider was used to send the position (latitude and longitude) of the vehicle from a remote place over the internet. GPRS was activated on this module using the HTTP protocol which sends the current GPS coordinates to the web server which was created using a Javascript. This feature adds to the safety of the bicycle. 


\section{H. Data Processing and Acquisition}

In this bicycle system the data processing was done in a distributive manner on two 8 bit $16 \mathrm{MHz}$ Atmega2560 microcontrollers. The drive microcontroller plans the motion for the bicycle using the algorithm fed to it and executes appropriate commands to the actuators. Along with this, the drive microcontroller also receives the GPS coordinates, SMS and call commands through the GSM Modem and controls the drive motor. The required steer angle for reaching the destination point using the path planning algorithm is sent to the steer microcontroller using UART communication.

\section{Power Distribution and Management}

Proper voltage regulator circuits were in place to provide different voltages to different components. The same is clear from the electronic architecture as shown in Fig.(10).

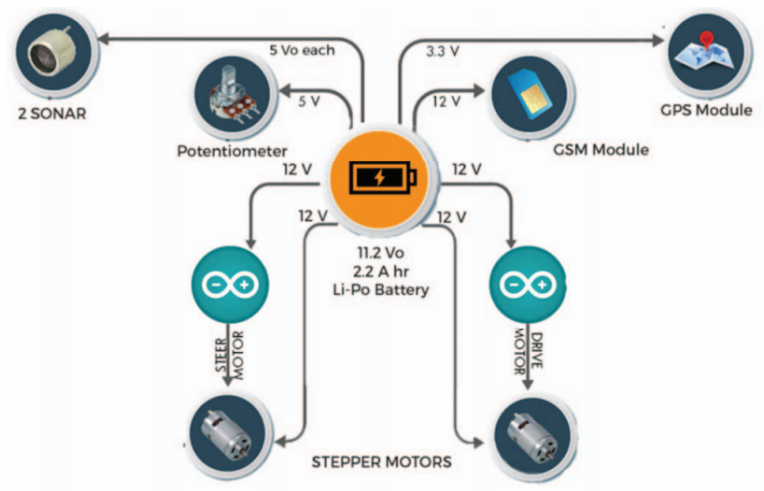

Fig. 10. Power management and electronic architecture

Each of the $12 \mathrm{~V}$ DC servo motors draws a current of $800 \mathrm{~mA}$ under no load condition and 7.5A current under the maximum speed condition. The i-Bike, being autonomous, uses various electronic components for its operation. So, a proper power distribution system was designed by taking into account the power ratings of all the electronic components used. The whole system of the i-Bike was powered using a single $11.7 \mathrm{~V} 2.2$ AH Li-polymer battery. In order to supply power to all kinds of components which differ in their voltage and power ratings proper voltage regulator circuits were designed.

The motor used in the model was a 12 volt and $7.5 \mathrm{amps}$ driven DC motor. The $11.7 \mathrm{~V}, 2200 \mathrm{mAH}$ LiPo battery [14] was close to the voltage rating and with a discharge current of $30 \times 2200 m A H=66 A m p$, it was a perfect choice. LiPo battery was preferred because of its light weight, high discharge rate and relatively good capacity. This battery also supports other components in the bike for power requirements, thus keeping a margin in Amps available and reducing the stress on the battery and increasing its life. Two or three mid to large capacity lithium batteries could easily fit on one ibike, giving potential ranges of 50 miles $(80 \mathrm{~km})$ or more. Well designed and soldered PCBs were used for all kinds of power distribution and signal conditioning circuits, further adding to the robustness of the vehicle. The table below summarizes the complete requirements.

\begin{tabular}{|c|c|c|}
\hline Components & Specifications & Description \\
\hline $\begin{array}{l}\text { Arduino } \\
\text { Mega[A] }\end{array}$ & $\begin{array}{l}\text { Input Voltage } \\
\text { (Recommended) - } 7 \text { to } 12 \mathrm{~V} \text {. } \\
\text { DC current per } \mathrm{I} / \mathrm{O} \text { pin - } 40 \\
\mathrm{~mA} \text {. } \\
\text { DC current for } 3.3 \mathrm{~V} \text { pin - } 50 \\
\mathrm{~mA}\end{array}$ & $\begin{array}{l}\text { It has a voltage } \\
\text { regulator installed on } \\
\text { the board. There are } \\
5 \mathrm{~V} \text { and } 3.3 \mathrm{~V} \text { pin } \\
\text { outs on the board that } \\
\text { supplies power to } \\
\text { other components. }\end{array}$ \\
\hline $\begin{array}{l}\text { DC Servo } \\
\text { Encoder } \\
\text { Motors[B] }\end{array}$ & $\begin{array}{l}\text { Input Voltage - } 12 \mathrm{~V} \text {. } \\
\text { No load current - } 800 \mathrm{~mA} \text {. } \\
\text { Load current - } 7.5 \mathrm{~A} \text { (max). } \\
\text { Pulse and Direction pins } \\
\text { Input Voltage - } 2.5,3.3 \text { or } 5 \\
\text { V. }\end{array}$ & $\begin{array}{l}\text { The motors are } \\
\text { powered separately } \\
\text { as they have high } \\
\text { power requirement. } \\
\text { Arduino controls the } \\
\text { speed and direction } \\
\text { of motor. }\end{array}$ \\
\hline $\begin{array}{l}\text { SIM900 } \\
\text { GSM } \\
\text { Module[C] }\end{array}$ & $\begin{array}{l}\text { Power supply - } 5 \mathrm{~V} \text {. } \\
\text { Current consumption } \\
\text { (continuous) - } 500 \mathrm{~mA} \text { (max) } \\
\text { Sleep Mode - } 1.5 \mathrm{~mA} \text {. }\end{array}$ & $\begin{array}{l}\text { The module has a DC } \\
\text { jack of } 12 \mathrm{~V} \text { with a } \\
\text { voltage regulator to } \\
\text { take down the } \\
\text { voltage to } 5 \mathrm{~V} \text {. }\end{array}$ \\
\hline $\begin{array}{l}\text { Sonar module } \\
\text { HC-SR04[D] }\end{array}$ & $\begin{array}{l}\text { Operating Voltage - } 5 \mathrm{~V} \text {. } \\
\text { Operating Current - } 15 \mathrm{~mA} \text {. }\end{array}$ & $\begin{array}{l}\text { It is a continuous } \\
\text { power consumption } \\
\text { source }\end{array}$ \\
\hline $\begin{array}{l}66 \text { channel } \\
\text { GPS } \\
\text { module[E] }\end{array}$ & $\begin{array}{l}\text { Operating Voltage }-3.3 \mathrm{~V} \text {. } \\
\text { Power Consumption - } 55 \mathrm{~mA} \\
\text { (acquisition), } 40 \mathrm{~mA} \\
\text { (tracking). }\end{array}$ & $\begin{array}{l}\text { Data output UART } \\
\text { interface } 3.3 \mathrm{~V}\end{array}$ \\
\hline
\end{tabular}

Fig. 11. Componenet specifications and power ratings

\section{TESTING AND REsults}
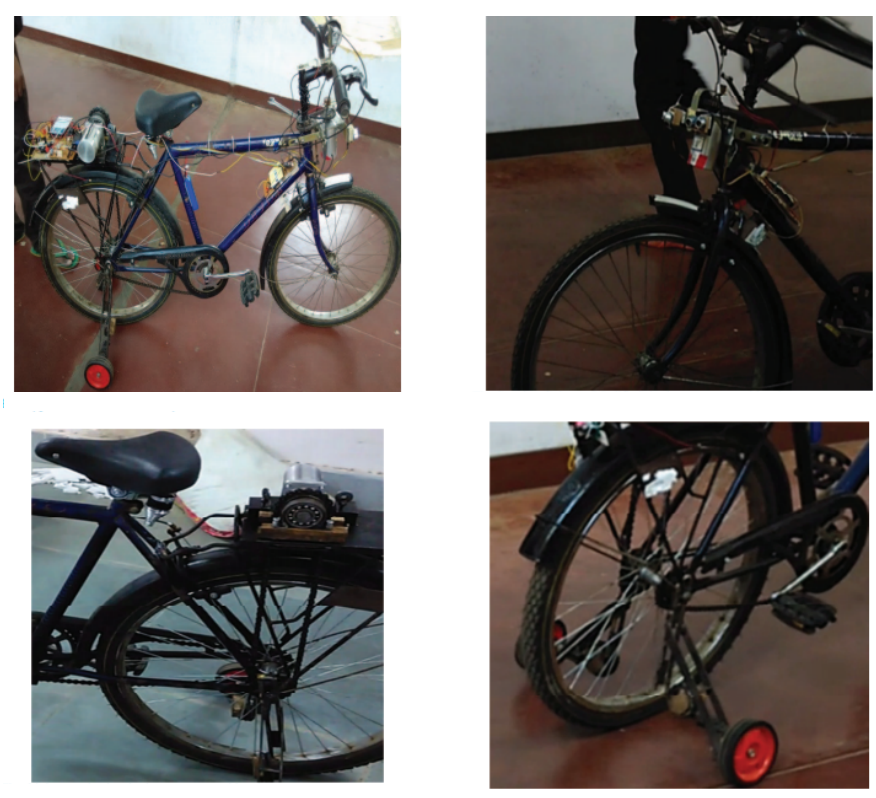

Fig. 12. Testing and Results for the working prototype of i-Bike 


\section{A. Application areas}

There are many applications of a smart and autonomous bicycle in today's world. It will be a boon for people who are differently-abled, people who are visually impaired or those who have difficulty in localization can use this vehicle to travel across a crowded city. With the autonomous ability of the bicycle comes the freedom for multiple people to use the same vehicle for transit. The bicycle can to rode to a place and then be called back to be rode to a different place, just by using mobile SMS (while maintain the security all along). The product can also be used to deliver courier or food to customers as it is capable to traversing through narrow and crowded lanes. With the ability to travel through narrow roads, it would be the ideal vehicle for Street view mapping. The painful task of street view mapping can be done by a smart and autonomous bicycle. It could also be used for real-time traffic monitoring as it wouldnt be adding to the traffic. A smart bicycle would also ease the pain of properly and systematically parking a bicycle as one wouldnt need to scout manually for an available parking space. As per need, the bicycle could be called to ones location instead of manually walking to the parking zone. In this modern era, we have huge factories with complex equipments; an autonomous bicycle would be the ideal mode of transport between any two points in the factory as it can then return to its designated space after assisting technicians move.

\section{B. Future prospects}

The algorithm used by the bicycle to achieve its autonomy can be used in ally form of autonomous vehicle especially autonomous Electric scooters. The engaging mechanism used for dual mode can be used in other vehicles where such need might arise. The MOC circuit used to ensure resetting of the steering even in the event of a mechanical offset can be used in other autonomous vehicles to ensure that the vehicle travels in a rectilinear fashion.

\section{LIMITATIONS AND CONCLUSIONS}

Current experiments with the prototype have shown obstacle avoidance capability with sonar at slow speeds, but further work must be done to avoid obstacles at higher speeds. The design of the spring limits the maximum speed during a turn and the maximum tilt angle which needs further optimizations. The design is also suitable for flat terrain with minor disturbances but some fluctuations still exist. For precise control of the bicycle the backlash error in the steering and rear wheel needs to be reduced and along with that the processing power could be increased.

The bicycle achieved what it set-out to, initially. It demonstrated great robustness as any user can sit on it to drive it anywhere and then the tests have shown that one SMS sent to the bicycle activates the autonomous mode and the bicycle avoids all obstacles in its way, giving the user a track of its position online, reaches the final destination.

\section{ACKNOWLEDGMENT}

The authors would like to thank the management committee of the Lal Bahadur Shastri Hall of Residence, IIT Kharagpur , along with the respected warden, Professor D. K. Maity for his kind assistance and encouragement. We would also like to thank our Institute authorities, the Gymkhana body (session 2014-15) and the Gymkhana President, IIT Kharagpur for providing us with this great opportunity to showcase our talent and skills. We would like to thank Mr. Jignesh Sindha, $\mathrm{PhD}$ scholar at IIT Kharagpur from the bottom of our hearts for his guidance and motivation throughout. Lastly, this project would never have been completed without Mr. Sanjay, bicycle mechanic whose technical knowledge about the little things in a bicycle came handy throughout our journey.

\section{REFERENCES}

[1] L.Keo, M.Yamakita, "Dynamic Model of a Bicycle with a balancer and its control Proceedings, Bicycle and Motorcycle Dynamics 2010 Symposium on the Dynamics and Control of Single Track Vehicles, 2022 October 2010, Delft, The Netherlands

[2] M. Yamakita, A. Utano and K. Sekiguchi, Experimental Study of Automatic Control of Bicycle with Balancer", IEEE/RSJ Int. Conf. on Intelligent Robots and Systems, Beijing, China, 2006, pp. 5606-5611.

[3] A. Murayama and M. Yamakita, Development of Autonomous Bike Robot with Balancer", SICE Annual Conference, Kagawa, Japan, 2007, pp. 1048-1052.

[4] L. Keo and M. Yamakita, Controller Design of an Autonomous Bicycle with Both Steering and Balancer Controls," IEEE Multiconference on Systems and Control. pp. 1294-1299, 2009.

[5] L. Keo and M. Yamakita,Controlling Balancer and Steering for Bicycle Stabilization," IEEE/RSJ Int. Conf. on Intelligent Robots and Systems. pp. 4541-4546, 2009.

[6] http://en.wikipedia.org/wiki/Bicycle_drivetrain_systems

[7] http://www.real-world-physics-problems.com/bicyclephysics.html

[8] https://sites.google.com/site/bikephysics/english-version/ 5-torques-acting-on-the-steering-axis

[9] Elbuluk, M.E.; Kankam, M.D. "Motor drive technologies for the powerby-wire (PBW) program: options, trends and tradeoffs" Aerospace and Electronic Systems Magazine, IEEE 1995

[10] Xiaolong Jin, Zhibao Su, Xijun Zhao, Jianwei Gong ”Design of a fuzzyPID longitudinal controller for Autonomous Ground Vehicle" 2011

[11] Ji Zhang, Sanjiv Singh, George Kantor "Robust Monocular Visual Odometry for a Ground Vehicle in Undulating Terrain"

[12] Keith W Gray, "Obstacle Detection and Avoidance for an autonomous farm tractor"

[13] Konyk, S., Jr."A student personal positioning system approach to the analysis, design and fabrication of actual control systems"

[14] http://www.ebikeschool.com/electric-bicycle-batteries-lithiumvs-lead-acid-batteries/ 